\title{
The Use Strategy of BIM Technology in Building Structure Design
}

\author{
Ying $\mathrm{Guo}^{1}$, Hao $\mathrm{Liu}^{2}$ \\ ${ }^{1}$ Dalian Vocational \&Technical College (DaLian Radio and TV University); Dalian, Liaoning Province, 116035 \\ ${ }^{2}$ China Construction Eighth Engineering Bureau Co., LTD. Northeast Company; Dalian, Liaoning Province, 116035
}

\begin{abstract}
This article analyzes the application functions of BIM technology. The research content of this article includes model integration functions, parameter editing functions, and information sharing functions. Combining the application value of BIM technology in architectural structure design, the author studies the key technical application points of designing visualized architectural models, design software selection, combing architectural structure performance, design parameter optimization processing, and structure collaborative design. The author's purpose is to improve the rationality of the building structure design plan and ensure the smooth progress of subsequent construction activities.
\end{abstract}

\section{Introduction}

The continuous maturity of building construction technology has improved the diversification of the content of building structure design in my country. At the same time, problems such as high engineering costs, low design efficiency, and poor professional collaboration are also becoming increasingly prominent. In particular, some large-scale engineering construction projects have a relatively large amount of preliminary building structure design. If the work cannot be completed efficiently and with high quality, it will also increase the time cost and economic cost, which will affect the economic benefits of the construction enterprise. The use of BIM technology has provided convenient conditions for the development of building structure design work to a large extent. Moreover, BIM technology can also provide timely feedback on existing problems with the help of 3D models, thereby reducing the time and cost of building structure design.

\section{Application Functions of BIM Technology}

\subsection{Model Integration Function}

Architectural designers use BIM technology to quantify various data and information in the entire building structure design process and elaborate on the parameters of each engineering design unit. Besides, BIM technology can aggregate various data information to construct a data information model covering various building structural elements. The integration of BIM models can be used to accurately display the positions of all components inside the building structure, such as walls, columns, beams, and other important supporting components of the house and their relationships. At the same time, it can not only truly and accurately reflect the specific structural features of the building design, but also use the physical information processing technology in the design system to perform dynamic simulation analysis of the design results. Using BIM technology, architectural designers can more intuitively and comprehensively understand the specific conditions of the building structure. In addition, BIM technology also helps builders to more accurately predict and judge the effect of building design to avoid designers making mistakes in building structure design and prevent safety accidents during building construction.

\subsection{Parameter Editing Function}

The basic content of BIM technology when used in building structure design is the construction of model database. In the process of database construction, the quantitative parameters formed by BIM technology are one of the very basic elements. It can be seen that in the process of building structure design, the parameter editing function is one of the important functions. The specific application process of its function is as follows. First, all parameter information needs to maintain a corresponding relationship with the relevant data in the three-dimensional model, which is conducive to the subsequent application analysis of the design model, thereby speeding up model error correction and correction. Second, the parameter content needs to be classified during parameter editing, which also requires the construction personnel to mark the parameter information in the design plan, thereby enhancing the application value of the parameter information.

\subsection{Information Sharing Function}

In the actual application process of BIM technology, in 
addition to the functional attributes mentioned in the first two items, it also has a good information sharing function. In the process of building structure modeling, designers can use model encapsulation to record relevant data in the building structure design drawing into the database. Moreover, when viewing the database information, it can be used as a whole to extract all the parameter information of the model [1]. In addition, with the help of network technology, other building structure design information can be integrated into the design. Simultaneously, BIM technology can also optimize the details of the structure according to the functionality of the designed building structure, and thereby improve the integrity of the design content of the building structure. Furthermore, in the context of the continuous improvement of computer technology and Internet of Things technology, the richness of BIM technology content is also increasing. In the meantime, the convenience of BIM technology for information sharing is constantly improving, thereby improving the compliance of structural design content.

\section{The Application Value of BIM Technology in Building Structure Design}

\subsection{Information Integration}

The total amount of data information and the types of information formats that need to be provided are also increasing when the scale of construction projects is being carried out in the context of continuous expansion of building structure design. Compared with the traditional two-dimensional data information, the format richness of data information is also increasing under the background of BIM technology application. Moreover, relying on information technology to smoothly transfer model data to the platform for display [2]. Technical personnel can directly perform information error correction on the platform, which achieves information integration compared with traditional cumbersome process BIM technology. From collection to model making, to data adjustment and modification, all can be completed on the platform. This also reduces the time consumption of $30 \%-50 \%$ in the design stage and improves the work efficiency of building structure design.

\subsection{Three-dimensional Design Content}

Judging from the current application situation, the BIM technology can not only transform the existing two-dimensional data information into three dimensions, but also build a three-dimensional structure model with the help of three-dimensional data information. In this way, the presentation effect of the design plan can be further improved, and the designer can evaluate the rationality of the design plan according to the model [3]. Moreover, the three-dimensional model can also be enlarged or reduced according to the scale. In this way, designers can view the details of the architectural design content and objectively evaluate the compliance of the design content such as the structure distribution law, the design of the through-wall pipes, and the reserved positions of the trunking. The compliance of many design contents can be evaluated based on the model established by BIM technology, so as to quickly optimize the design structure contents.

\subsection{Design Parameter Adjustment}

As mentioned in the previous chapters, the BIM technology can not only transform the existing two-dimensional data information into three dimensions, but also build a three-dimensional structure model with the help of three-dimensional data information. Designers can view the details of the structure with the help of scaling to evaluate the compliance of existing design parameters [4]. In the research process, if the corresponding problem is found, the designer can adjust the parameter information on the platform and regenerate the $3 \mathrm{D}$ data model after completing the data update. After that, the evaluation results were analyzed again, and the design parameters of the structure were gradually adjusted until a design scheme with higher reliability was obtained. This also reduces the time for re-adjusting the design plan by $60 \%-70 \%$, and improves the reliability of the design parameter content.

\section{The Application Points of BIM Technology in Building Structure Design}

\subsection{Design A Visual Architectural Model}

\subsubsection{Clear Modeling Principles}

The first task in the process of building structure design is to clarify the modeling principles. The specific content is as follows. First, the model established by BIM technology needs to be completely consistent with the building structure design model. Especially important structures should not have structural errors. Second, in order to facilitate data analysis, BIM models need to be built in stages. This is conducive to the rapid progress of the analysis work and also improves the efficiency of the design phase. Third, select the professional component types as shown in Figure 1, including "columns", "beams", and "plates". Fourth, the coordinate parameters of the model are easily not allowed to be changed, which facilitates the smooth calculation of other coordinates. Fifth, the content contained in the BIM model needs to be complete. It cannot have unrelated or incomplete structure. Otherwise, the naming principle needs to follow the building design code to improve the rationality of the design content [5]. 


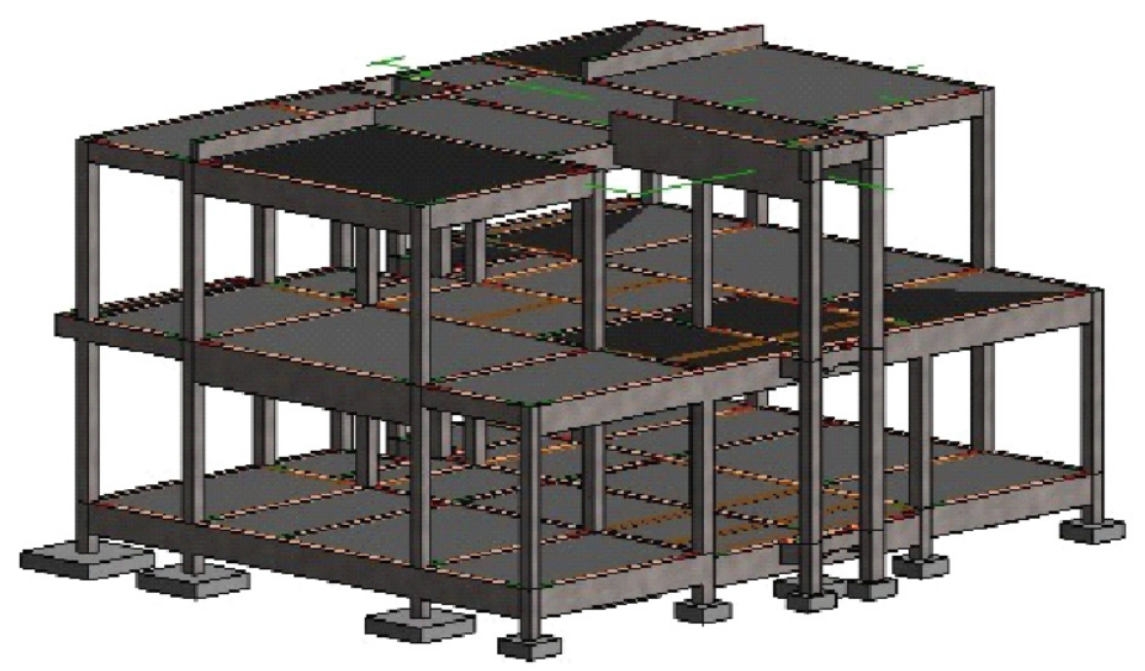

Figure 1 Schematic Diagram of BIM Structure Model

\subsubsection{Application Mode Selection}

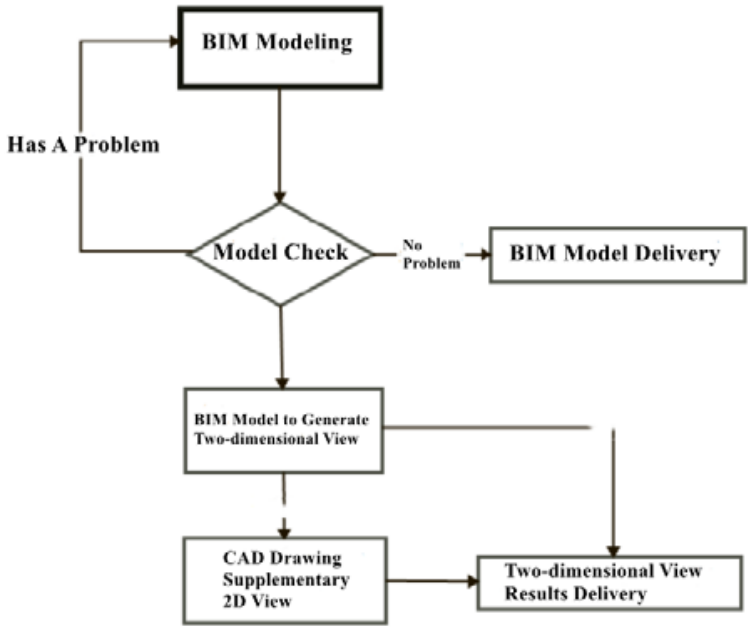

Figure 2 Schematic Diagram of BIM Technology Application Mode

Combining the current application experience, we can understand that the most commonly used mode when incorporating BIM technology into building structure design is to combine BIM technology and two-dimensional views. The specific application process is shown in Figure 2. In the application process of architectural structure design, BIM technology will be used to complete the formation of 3D models, and at the same time, $2 \mathrm{D}$ views will be exported. In the design of building structures, there will be some structures that do not conform to the domestic drawing habits, and these structures cannot be used to completely show the two-dimensional design drawings using BIM technology. At this time, designers need to use CAD software to supplement the missing content to improve the integrity of the design content. CAD software is also a relatively mature application mode, which is suitable for most building structure designs [6].

\subsubsection{Quality Management after Modeling}

After modeling the building structure, its quality management has gone through three application stages. The first stage is in the conceptual design stage, and its quality management focuses on checking the structural integrity and the compliance of the main component layout. The second stage is in the preliminary design stage. The focus of quality management is to verify the application form of the structure, the size of the main components, the layout of the superstructure, the design position of the structure node, the distribution of building structure units, and the location of standard floors. The third stage is in the construction drawing design stage, and its quality management focuses on the verification of deeper structure information. The verification content includes the specific parameter information of the component, reinforcement information, floor component parameter information, reserved structure size, reserved cavity position parameter, etc. [7].

\subsection{Choice of Design Software}

BIM technology is a collection of multiple types of technology, and more drawing software will be used during the application of the technology. Therefore, the choice of BIM technology software is also one of the very important contents. In the specific selection, the selected software needs to meet the following conditions. First, it has strong applicability to meet the requirements of the construction industry. And they also need to have a certain penetration rate to ensure the proficiency of personnel [8]. Second, it has certain functional characteristics, including data format conversion, secondary function expansion, information storage and other functions. Third, the economy is relatively high, the purchase cost is at a medium level, and the price is relatively high. Fourth, according to the operation level of the technicians, choose the matching design software to improve the rationality of the design content. 


\subsection{Sort out Building Structure Performance}

When designing a building structure, clarifying the performance of the building structure is also a very core content. The analysis result of this content also directly determines the combination of building structure. At the same time, the structural load performance is evaluated so that it can meet the established application effect [9]. Under the background of BIM technology application, all application data of construction projects can be imported into the analysis system, and accurate and reliable application data can be obtained after the analysis and processing of digital technology and big data technology. Besides, in this way, it can also be technically evaluated to obtain an application plan that meets the design requirements of the construction project, thereby improving the accuracy of the structural performance analysis results of the construction project. Moreover, the design of other structural contents is completed according to specific application requirements, which improves the reliability and effectiveness of the design results.

\subsection{Design Parameter Optimization}

In the case of BIM technology application, the very basic work content is to optimize the design of design parameters. In the specific work process, we can first

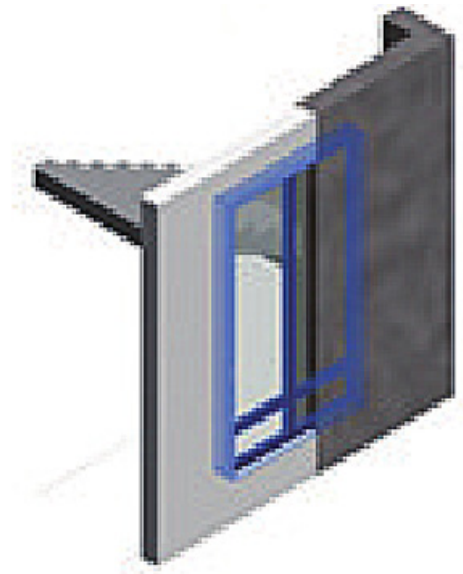

Before Collision Optimization complete the establishment of the three-dimensional structure model. Designers can view the details of the structure with the help of scaling, mark some disputed parameters, and adjust the optimizable parameters after discussion and calculation. Subsequently, the designer can also regenerate the three-dimensional data model, analyze the evaluation results again, and evaluate the cohesion of each link of the building structure. In this way, a reliable design plan is formed and its application value is improved [10].

\subsection{Co-design of Structure}

The main purpose of the structural system design is to match and analyze the correlation between the branch structures in the construction drawings, so that the collocation of various parameters can better meet the application requirements. In practical applications, BIM technology will import the basic parameter data of construction projects into the software, plan to build the corresponding structural model, and use the collision experiment to verify the conflict between the various structural models [11]. As shown in Figure 3, the BIM technology found that the building doors, windows and wall parameters conflicted in the collision experiment. After adjusting the parameters in time, give up the shelter position so that it can meet the requirements of construction specifications and avoid rework problems.

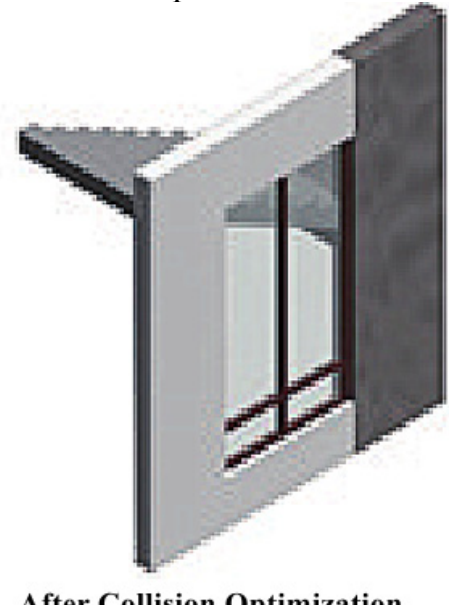

After Collision Optimization

Figure 3 Schematic Diagram of Collision Model

\section{Conclusion}

In summary, judging from the application status of BIM technology in building structure design, this technology can meet the overall requirements of building design and project construction. However, BIM technology also puts forward higher requirements on the professional skills and professionalism of designers and construction personnel. Therefore, construction companies should start with the grassroots staff, do a good job of personnel training, improve the management system, improve the application level of BIM technology, and promote faster and better development of the company.

\section{Acknowledgement}

2018 Science and Technology Innovation Project of Dalian Vocational \& Technical College

2019 Scientific Research and Innovation Fund Program of Dalian Vocational and Technical College

\section{References:}

1. Li Xiaoyin. Research on the application of BIM technology in architectural engineering structure design[J]. Science and Technology Innovation and Application, 2020(26): 91-92.

2. Wang Lei. Analysis of the application of BIM 
technology in the structural design of building engineering $[\mathrm{J}]$. Development of Building Technology, 2020, 47(15): 14-15.

3. Xi Xinyuan. Application of BIM technology in building structure design[J]. Engineering Technology Research, 2020, 5(15): 201-202.

4. Li Guanglong. Analysis of the application and practice of BIM technology in building structure design[J]. Engineering Construction and Design, 2020(15): 139-141.

5. Yin Junwen, Wu Zhenzhen, Li Yuxuan, Fu Rui. The application of BIM technology in the architectural design of Baishishan Geological Museum[J]. Housing and Real Estate, 2020(21): 73-74.

6. Qin Xiyang. On the effective application of BIM technology in building structure design[J]. Building Materials and Decoration, 2020(21): 94+97.

7. Zhou Shuyang. Research on the application of BIM technology in prefabricated building structure design[J]. Chinese and foreign entrepreneurs, 2020(20): 116.

8. Sun Bing. Application of BIM technology in prefabricated building structure design[J]. Building Structure, 2020, 50(13): 160.

9. Zhao Zhiqiang. Research on the application of BIM technology in building structure design[J]. Engineering Technology Research, 2020, 5(12): $199-200+202$.

10. He Kuang. The application of BIM technology in building structure design[J]. Engineering Technology Research, 2020, 5(12): 203-204.

11. Xue Zuwei. Research on the application of BIM technology in prefabricated building structure design[J]. Adhesion, 2020, 42(06): 105-108. 